\title{
High-precision $\alpha_{\mathrm{s}}$ from $\mathrm{W}$ and $\mathrm{Z}$ hadronic decays
}

\author{
David d'Enterria* \\ CERN, EP Department, CH-1211 Geneva 23, Switzerland \\ E-mail: David.d'Enterria@cern.ch
}

The extraction of the QCD coupling $\alpha_{\mathrm{s}}$ from the comparison of experimental data on inclusive $\mathrm{W}$ and $\mathrm{Z}$ bosons hadronic decays to state-of-the-art perturbative $\mathrm{QCD}$ calculations is reviewed. The relatively small amount of $\mathrm{W}$ data from $e^{+} e^{-} \rightarrow \mathrm{W}^{+} \mathrm{W}^{-}$collisions at LEP leads today to an non-competitive extraction of the strong coupling at the $\mathrm{Z}$ mass from the measured $\mathrm{R}_{\mathrm{W}}$ ratio of hadronic-to-leptonic branching fractions, $\alpha_{\mathrm{s}}\left(m_{\mathrm{Z}}\right)=0.117 \pm 0.042_{\exp } \pm 0.004_{\text {th }} \pm 0.001_{\text {par }}$ with a $\sim 35 \%$ propagated experimental uncertainty. Analysis of the much more abundant hadronic results at the $\mathrm{Z}$ pole leads to $\alpha_{\mathrm{s}}\left(m_{\mathrm{Z}}\right)=0.1203 \pm 0.0030$, with a $2.5 \%$ uncertainty by combining three different pseudo-observables (ratio of hadronic-to-leptonic widths $\mathrm{R}_{\mathrm{Z}}$, hadronic peak cross section $\sigma_{\mathrm{Z}}^{\text {had }}$, and total width $\Gamma_{\mathrm{Z}}^{\mathrm{tot}}$ ). An $\alpha_{\mathrm{s}}$ determination with per mille uncertainty requires highstatistics $\mathrm{W}$ and $\mathrm{Z}$ bosons data samples at future $e^{+} e^{-}$colliders, such as the FCC-ee, combined with even higher precision $\left(\mathrm{N}^{4} \mathrm{LO}\right) \mathrm{pQCD}$ calculations.

$\alpha_{s}(2019):$ Workshop on precision measurements of the QCD coupling constant 11-15 February, 2019

Trento, Italy

${ }^{*}$ Speaker. 


\section{Introduction}

The strong coupling $\alpha_{\mathrm{s}}$ is one of the fundamental parameters of the Standard Model (SM), and its value not only directly affects the stability of the electroweak vacuum [1] but it chiefly impacts the theoretical calculations of all scattering and decay processes involving real and/or virtual quarks and gluons [2]. Known today with a $0.9 \%$ precision, $\alpha_{\mathrm{s}}$ is the worst known of all fundamental interaction couplings in nature [3], and such an imprecision propagates as an input parametric uncertainty in the calculation of many important physics observables, in particular in the electroweak (EW), Higgs, and top-quark SM sectors [4]. The current world-average value, $\alpha_{\mathrm{s}}\left(m_{\mathrm{z}}\right)=0.1181 \pm 0.0011$ [3], is derived from a combination of six subclasses of approximatelyindependent observables measured in $e^{+} e^{-}$collisions (hadronic $\mathrm{Z}$ boson and $\tau$ decays, plus event shapes and jet rates), deep-inelastic scattering DIS (structure functions and global fits of parton distributions functions PDFs), and p-p collisions (inclusive top-pair cross sections), as well as from lattice QCD computations constrained by the empirical values of hadron masses and decay constants. In order to be combined into the $\alpha_{\mathrm{s}}\left(m_{\mathrm{z}}\right)$ world-average, the experimental (or lattice) results need to have a counterpart perturbative $\mathrm{QCD}(\mathrm{pQCD})$ prediction at next-to-next-to-leadingorder NNLO (or beyond) accuracy.

In principle, among the theoretically and experimentally "cleanest" $\alpha_{\mathrm{s}}$ extractions are those based on the hadronic decays of electroweak bosons. This is so because (i) the inclusive hadronic $\mathrm{W}$ and $\mathrm{Z}$ decays can be very accurately measured in $e^{+} e^{-}$collisions provided one has large enough data samples, (ii) the corresponding theoretical predictions can be computed with a very high theoretical accuracy, today up to $\mathscr{O}\left(\alpha_{\mathrm{s}}^{4}\right)$, i.e. $\mathrm{N}^{3} \mathrm{LO}$, in pQCD [5], plus mixed $\mathscr{O}\left(\alpha \alpha_{\mathrm{s}}\right)$ pQCD-EW [6, 7] and (in the $\mathrm{Z}$ case) the full two-loop $\mathscr{O}\left(\alpha^{2}\right)$ EW corrections [8], and (iii) non-pQCD effects are suppressed thanks to the large energy scale given by the electroweak masses $\left(m_{\mathrm{W}, \mathrm{Z}} \gg \Lambda_{\mathrm{QCD}} \approx\right.$ $0.2 \mathrm{GeV}$ ). The common high-precision hadronic observables used to extract $\alpha_{\mathrm{s}}$ in $e^{+} e^{-}$annihilation at the $\mathrm{W}$ and $\mathrm{Z}$ boson masses can be schematically decomposed as follows:

- total $\mathrm{W}$ and $\mathrm{Z}$ hadronic width:

$$
\begin{aligned}
\Gamma_{\mathrm{W}, \mathrm{Z}}^{\mathrm{had}}(Q) & =\frac{\sigma\left(e^{+} e^{-} \rightarrow(\mathrm{W}, \mathrm{Z}) \rightarrow \text { hadrons }\right)}{\sigma\left(e^{+} e^{-} \rightarrow(\mathrm{W}, \mathrm{Z}) \rightarrow \mathrm{X}\right)} \\
& =\Gamma_{\mathrm{W}, \mathrm{Z}}^{\mathrm{Born}}\left(1+\sum_{i=1}^{4} c_{i}(Q)\left(\frac{\alpha_{\mathrm{s}}(Q)}{\pi}\right)^{i}+\mathscr{O}\left(\alpha_{\mathrm{s}}^{5}\right)+\delta_{\mathrm{EW}}\left(\alpha, \alpha^{2}\right)+\delta_{\mathrm{m}}\left(\alpha \alpha_{\mathrm{s}}\right)+\delta_{\mathrm{np}}\right)
\end{aligned}
$$

where the Born width $\Gamma_{\mathrm{W}, \mathrm{Z}}^{\mathrm{Born}}=f\left(G_{F}, N_{C}, m_{\mathrm{W}, \mathrm{Z}}^{3} ; \sum\left|\mathrm{V}_{\mathrm{ij}}\right|^{2}\right)$ depends on the Fermi constant $G_{F}$ and the number of colours $N_{C}$, and in the $\mathrm{W}$ case on the sum of CKM matrix elements $\left|\mathrm{V}_{\mathrm{ij}}\right|^{2}$, and

- ratio of inclusive hadronic-to-leptonic widths (that commonly includes also the $\tau$ lepton, which proceeds via offshell $\mathrm{W}$ decays):

$$
\begin{aligned}
\mathrm{R}_{\tau, \mathrm{W}, \mathrm{Z}}(Q) & =\frac{\sigma\left(e^{+} e^{-} \rightarrow(\tau, \mathrm{W}, \mathrm{Z}) \rightarrow \text { hadrons }\right)}{\sigma\left(e^{+} e^{-} \rightarrow(\tau, \mathrm{W}, \mathrm{Z}) \rightarrow \ell^{+} \ell^{-}\right)} \\
& =\mathrm{R}_{\tau, \mathrm{W}, \mathrm{Z}}^{\mathrm{EW}}\left(\alpha, \alpha^{2} ; Q\right)\left(1+\sum_{i=1}^{4} c_{i}(Q)\left(\frac{\alpha_{\mathrm{s}}(Q)}{\pi}\right)^{i}+\mathscr{O}\left(\alpha_{\mathrm{s}}^{5}\right)+\delta \mathrm{m}\left(\alpha \alpha_{\mathrm{s}}\right)+\delta_{\mathrm{np}}\right)
\end{aligned}
$$


where the $\mathrm{R}_{\tau, \mathrm{W}, \mathrm{Z}}^{\mathrm{EW}}$ prefactor accounts for the purely electroweak dependence of the ratio.

In both expressions (1) and (2), $Q=m_{\tau}, m_{\mathrm{W}}, m_{\mathrm{Z}}$ is the relevant momentum transfer in the process, $c_{i}$ are coefficients of the pQCD expansion calculated today up to a finite order $i=4$, the $\mathscr{O}\left(\alpha_{\mathrm{s}}^{5}\right)$ term indicates (sub-permille) corrections at $\mathrm{N}^{4} \mathrm{LO}$ accuracy not yet computed, and $\delta_{\mathrm{m}}\left(\alpha \alpha_{\mathrm{s}}\right)$ and $\delta_{\mathrm{np}}\left(\Lambda_{\mathrm{QCD}}^{p} / Q^{p}\right)$ correspond to mixed pQCD-EW and power-suppressed non-perturbative corrections, respectively. It is important to note that the Born level term in the calculation of $\mathrm{W}$ and $\mathrm{Z}$ hadronic decays is completely independent of the QCD coupling, and that all $\alpha_{\mathrm{s}}$ sensitivity comes through (small) higher-order loop corrections. Indeed, for $\alpha_{\mathrm{s}}\left(m_{\mathrm{z}}\right) \approx 0.118$, the size of the QCD sum in Eq. (2) amounts to a $\sim 3 \%$ effect in the calculation of $\mathrm{R}_{\mathrm{W}, \mathrm{Z}}$, and thereby at least permille measurement accuracies in this ratio are required for a competitive $\alpha_{\mathrm{s}}\left(m_{\mathrm{z}}\right)$ determination. Such an experimental precision has been achieved in $\tau$ and $\mathrm{Z}$ boson measurements, but not in the $\mathrm{W}$ boson case, and that is why the latter does not yet provide a precise $\alpha_{\mathrm{s}}$ extraction [9] as discussed below. Reaching permille uncertainties in $\alpha_{\mathrm{s}}$ determinations requires many orders of magnitude smaller uncertainties in the experimental $\tau, \mathrm{W}$ and $\mathrm{Z}$ measurements than today, a situation only reachable at a future $e^{+} e^{-}$collider such as the FCC-ee [10] (or before, at B-factories, for the $\tau$ lepton).

It is instructive to consider the $\alpha_{\mathrm{s}}$ extraction via $\tau$ lepton decays using Eq. (2), which proceeds via offshell $\mathrm{W}$ hadronic decays (involving only the kinematically allowed $u, d$, and $s$ quarks), before studying the (onshell) electroweak bosons case. In this case, the ratio of hadronic to leptonic decays, known experimentally to within $\pm 0.23 \%, \mathrm{R}_{\tau, \exp }=3.4697 \pm 0.0080$, yields $\alpha_{\mathrm{s}}\left(m_{\mathrm{z}}\right)=$ $0.1192 \pm 0.0018$ with a $1.5 \%$ uncertainty, through a combination of results from different $\mathrm{N}^{3} \mathrm{LO}$ calculations (contour-improved CIPT, and fixed-order FOPT, perturbation theory) with different treatments of the non-pQCD corrections [11, 12]. The non-perturbative power-suppressed $\delta_{\text {np }}$ term in Eq. (2) is $\mathscr{O}\left(\Lambda_{\mathrm{QCD}}^{2} / m_{\tau}^{2}\right) \approx 10^{-2}$, and thereby not negligible at variance with the much heavier $\mathrm{W}$ and $\mathrm{Z}$ bosons case. Reducing the current $\alpha_{\mathrm{s}}\left(m_{\mathrm{Z}}\right)$ extraction uncertainties from the $\tau$ lepton requires controlling the non-pQCD uncertainties through better experimental data (in particular, $\tau$ spectral functions) than those from ALEPH and OPAL currently available (e.g., from B-factories now, and FCC-ee in the future) [12], solving CIPT-FOPT discrepancies, and eventually extending the calculations to $\mathrm{N}^{4} \mathrm{LO}$ accuracy.

\section{Extraction of $\alpha_{\mathrm{s}}\left(m_{\mathrm{Z}}\right)$ from hadronic $\mathbf{W}$ decays}

The current state-of-the-art calculations of $\mathrm{W}$ boson hadronic decays include $\mathrm{N}^{3} \mathrm{LO}$ pQCD [5], one-loop $\mathscr{O}(\alpha)$ EW [13], and mixed two-loop $\mathscr{O}\left(\alpha \alpha_{\mathrm{s}}\right)$ pQCD-EW [6] corrections. Numerically, the relative weights of the different terms appearing in Eqs. (1) and (2) amount to [9]: $\Gamma_{\mathrm{W}}^{\text {Born }}$, $\mathrm{R}_{\mathrm{W}}^{\mathrm{EW}} \approx 96.6 \%, \mathscr{O}\left(\alpha_{\mathrm{s}}^{1}\right) \approx 3.7 \%, \mathscr{O}\left(\alpha_{\mathrm{s}}^{2}\right) \approx 0.2 \%, \mathscr{O}\left(\alpha_{\mathrm{s}}^{3}\right) \approx-0.1 \%, \mathscr{O}\left(\alpha_{\mathrm{s}}^{4}\right) \approx-0.02 \%, \mathscr{O}(\alpha) \approx$ $-0.35 \%, \delta_{\mathrm{m}} \approx-0.05 \%$, with negligible $\delta_{\mathrm{np}}$ non-pQCD effects, suppressed by $\mathscr{O}\left(\Lambda_{\mathrm{QCD}}^{4} / \mathrm{m}_{\mathrm{W}}^{4}\right)$ power corrections. However, the calculations suffer from a significant parametric uncertainty from the input CKM matrix elements. Indeed, the Born-level $\mathrm{W}$ hadronic decay width is directly proportional to the sum over the first two rows of the CKM matrix, $\Gamma_{\mathrm{W}}^{\mathrm{Born}} \propto \sum_{\mathrm{u}, \mathrm{c}, \mathrm{d}, \mathrm{s}, \mathrm{b}}\left|V_{\mathrm{ij}}\right|^{2}$ (the top quark is kinematically forbidden in $\mathrm{W}$ decays), whose uncertainty is dominated by the $1.6 \%$ imprecision of the measured charm-strange quark mixing element, $\left|V_{\mathrm{cs}, \exp }\right|=0.986 \pm 0.016$ [3]. Thus, using the experimental CKM elements, the prefactor $\sum_{\mathrm{u}, \mathrm{c}, \mathrm{d}, \mathrm{s}, \mathrm{b}}\left|V_{\mathrm{ij}}\right|^{2}=2.024 \pm 0.032$ propagates as a final $1.6 \%$ 
uncertainty into any hadronic $\mathrm{W}$ decay calculation today. In order to assess the impact of such a parametric uncertainty, one can impose CKM unitarity and take $\sum_{\mathrm{u}, \mathrm{c}, \mathrm{d}, \mathrm{s}, \mathrm{b}}\left|V_{\mathrm{ij}}\right|^{2} \equiv 2$.

Unfortunately, on the experimental side the situation is even much less precise. The relevant $\mathrm{LEP}^{+} \mathrm{W}^{-}$data are statistically poor, based on about $5 \cdot 10^{4} \mathrm{~W}$ bosons alone, and the associated extraction of $\alpha_{\mathrm{s}}\left(m_{\mathrm{z}}\right)$ is truly non-competitive today. From the current value of the W hadronic width, $\Gamma_{\mathrm{W}}^{\text {had,exp }}=\Gamma_{\mathrm{W}}^{\text {tot,exp }} \cdot \mathscr{B}_{\mathrm{W}}^{\text {had, } \exp }=1405 \pm 29 \mathrm{MeV}$ with a $2 \%$ uncertainty [3], one can barely constraint the QCD coupling: $\alpha_{\mathrm{s}}\left(m_{\mathrm{z}}\right)=0.069 \pm 0.065_{\exp } \pm 0.050_{\mathrm{par}}$, or assuming CKM unitarity, $\alpha_{\mathrm{s}}\left(m_{\mathrm{z}}\right)=0.107 \pm 0.066_{\mathrm{exp}} \pm 0.002_{\mathrm{par}} \pm 0.001_{\text {th }}$. If one uses, instead, the value of the hadronic/leptonic ratio experimentally known with a $1.2 \%$ precision $\left(\mathrm{R}_{\mathrm{W}}^{\exp }=2.068 \pm 0.025\right)$, one obtains $\alpha_{\mathrm{s}}\left(m_{\mathrm{z}}\right)=0.00 \pm 0.04_{\mathrm{exp}} \pm 0.16_{\mathrm{par}}$ (with the experimental CKM matrix) or $\alpha_{\mathrm{s}}\left(m_{\mathrm{z}}\right)=0.117 \pm$ $0.042_{\mathrm{exp}} \pm 0.004_{\text {th }} \pm 0.001_{\mathrm{par}}$ (assuming CKM unitarity) [9]. This last value shows that, in the best scenario, the derived $\alpha_{\mathrm{s}}\left(m_{\mathrm{z}}\right)$ value has currently a huge $\pm 36 \%$ propagated uncertainty (Fig. 1, left).
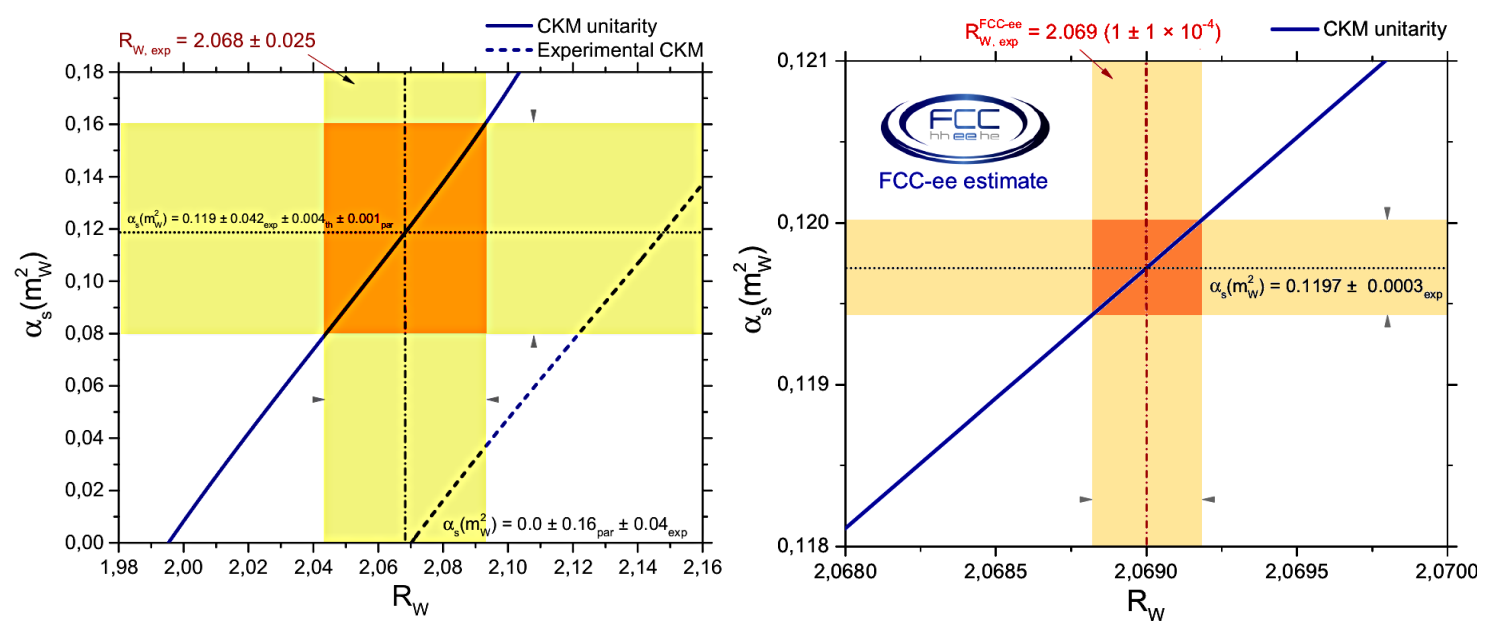

Figure 1: Extraction of $\alpha_{\mathrm{s}}$ from the hadronic/leptonic $\mathrm{W}$ decay ratio $\mathrm{R}_{\mathrm{W}}$, using the current data (left) and expected at the FCC-ee with experimental uncertainties alone (right) [9]. Note the wildly different $x$ - and $y$-axes scales. The diagonal blue line in both plots assumes CKM matrix unitarity.

At the FCC-ee, the total $\mathrm{W}$ width $\Gamma_{\mathrm{W}}^{\text {tot }}$ can be accurately measured through a threshold $e^{+} e^{-} \rightarrow$ $\mathrm{W}^{+} \mathrm{W}^{-}$scan around $\sqrt{\mathrm{s}}=2 m_{\mathrm{W}}$, and also the $\mathrm{R}_{\mathrm{W}}$ ratio will profit from the huge sample of $5 \cdot 10^{8} \mathrm{~W}$ bosons (a thousand times more than those collected at LEP) thereby reducing the statistical uncertainty of $\mathrm{R}_{\mathrm{W}}$ to around $0.005 \%$. Neglecting parametric uncertainties, the high-precision $\mathrm{W}$ decay measurements at the FCC-ee would significantly improve the extraction of $\alpha_{\mathrm{s}}$ with propagated experimental uncertainties of order $0.4 \%$. A value that could be further reduced to $\sim 0.2 \%$ through the measurement of the $\mathrm{R}_{\mathrm{W}}$ ratio in three $e^{+} e^{-} \rightarrow \mathrm{W}^{+} \mathrm{W}^{-}$final states ( $\ell v \ell v, \ell v \mathrm{qq}$, qqqq), and/or combining it with the $\alpha_{\mathrm{s}}$ value derived from the total width $\Gamma_{\mathrm{W}}^{\mathrm{tot}}$. Indeed, the ratio of cross sections $\sigma(\mathrm{WW} \rightarrow \mathrm{qq} q \mathrm{qq}) / \sigma(\mathrm{WW} \rightarrow \ell v \ell v)$ is proportional to $\left(\mathrm{R}_{\mathrm{W}}\right)^{2}$, thereby gaining a factor two in statistical sensitivity, and being totally independent of potential modifications of the weak coupling running as well as free from cross section normalization uncertainties [10]. Figure 1 (right) shows the estimated $\alpha_{\mathrm{s}}$ extraction from the expected improved measurement of $\mathrm{R}_{\mathrm{W}}$ at FCC-ee, assuming that $\mathrm{V}_{c s}$ has a negligible uncertainty (or, identically, assuming CKM matrix unitarity). A full determination of $\alpha_{\mathrm{s}}$ with permille uncertainty including also parametric and theoretical uncertainties 
will require two more developments: (i) a significantly reduced uncertainty of the $\mathrm{V}_{c s}$ CKM element, and (ii) computing the $\mathrm{N}^{4} \mathrm{LO}$ pQCD term $\mathscr{O}\left(\alpha_{\mathrm{s}}^{5}\right)$, as well as missing two-loop electroweak corrections (available now for the $\mathrm{Z}$ boson) of Eqs. (1) and (2).

\section{Extraction of $\alpha_{\mathrm{s}}\left(m_{\mathrm{Z}}\right)$ from hadronic $\mathrm{Z}$ decays}

On the theory side, the current state-of-the-art $\mathrm{Z}$ boson hadronic decays calculations include $\mathrm{N}^{3} \mathrm{LO}$ pQCD [5], plus full two-loop $\mathscr{O}\left(\alpha^{2}\right)$ EW, and mixed two-loop $\mathscr{O}\left(\alpha \alpha_{\mathrm{s}}\right)$ pQCD-EW corrections (see Ref. [8] for a complete list of relevant references). Numerically, the size of the Born term appearing in Eqs. (1) and (2) is $\Gamma_{\mathrm{Z}}^{\mathrm{Born}}, \mathrm{R}_{\mathrm{Z}}^{\mathrm{EW}} \approx 96.8 \%$, and one can see again that the $\alpha_{\mathrm{s}}$ dependence on these observables only enters through (small) higher-order corrections. However, as for the W boson case, the non-perturbative effects encoded in the $\delta_{\mathrm{np}}$ term are power-suppressed by $\mathscr{O}\left(\Lambda_{\mathrm{QCD}}^{4} / m_{\mathrm{Z}}^{4}\right)$. The current QCD coupling extraction based on $\mathrm{Z}$ hadronic decays uses not just $\Gamma_{\mathrm{Z}}^{\text {tot,exp }}$ (yielding $\alpha_{\mathrm{s}}\left(m_{\mathrm{Z}}\right)=0.1209 \pm 0.0049$ ) and $\mathrm{R}_{\mathrm{Z}}^{\text {exp }}$ (giving $\alpha_{\mathrm{s}}\left(m_{\mathrm{Z}}\right)=0.1237 \pm 0.0043$ ), but also the hadronic peak cross section $\sigma_{Z}^{\text {had,exp }}=12 \pi / m_{Z} \cdot \Gamma_{Z}^{e} \Gamma_{Z}^{\text {had }} /\left(\Gamma_{Z}^{\text {tot }}\right)^{2}=41.540 \pm 0.037 \mathrm{nb}$, all measured at LEP with a data sample of $1.7 \cdot 10^{7} \mathrm{Z}$ bosons [14], to derive $\alpha_{\mathrm{s}}\left(m_{\mathrm{Z}}\right)=0.1203 \pm 0.0030$ with a $2.5 \%$ uncertainty [15] (the extraction based on LEP-only data is $\alpha_{\mathrm{s}}\left(m_{\mathrm{Z}}\right)=0.1221 \pm 0.0031$
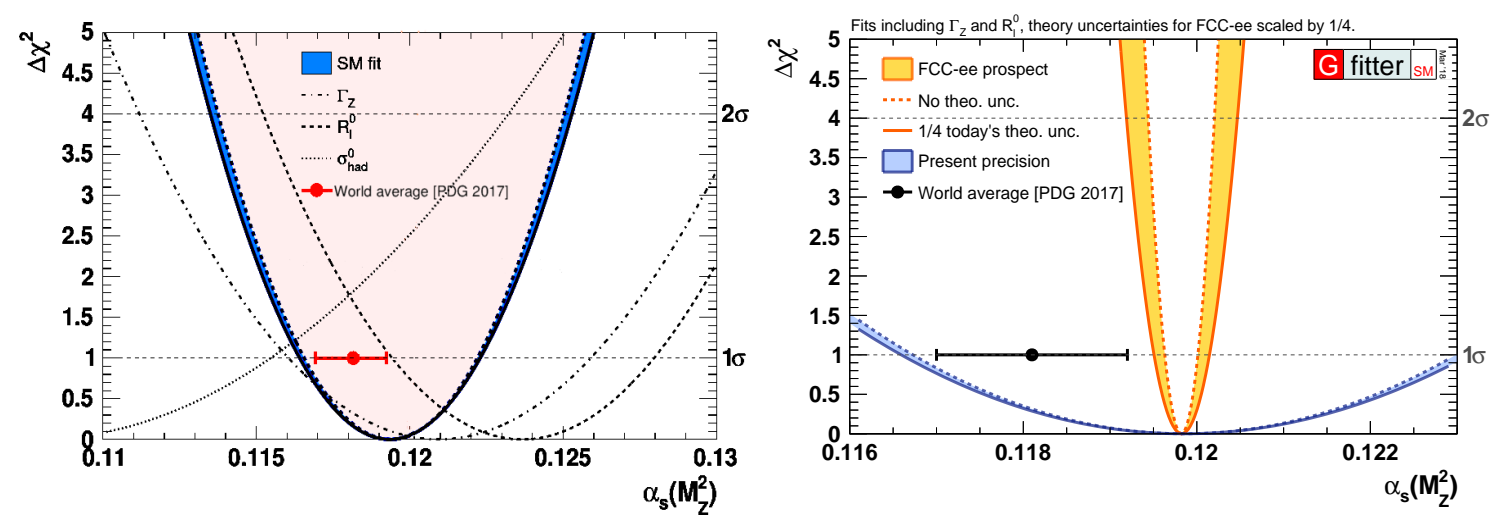

Figure 2: Extracted $\alpha_{\mathrm{s}}$ values from hadronic $\mathrm{Z}$ decay data compared to the current world-average (circle). Left: Using the current experimental measurements of $\Gamma_{Z}^{\text {tot }}$ (dashed-dotted), $\mathrm{R}_{\mathrm{Z}}$ (dashed), and $\sigma_{\mathrm{Z}}^{\text {had }}$ (dotted lines). Right: Expected at the FCC-ee from $\Gamma_{\mathrm{Z}}^{\text {tot }}$ and $\mathrm{R}_{\mathrm{Z}}$ (yellow band) without theoretical uncertainties (dotted curve) and with the current ones divided by a factor of four (solid curve). The blue band in both plots shows the result of the full SM electroweak fit today [15].

as quoted in the Electroweak chapter of the PDG [3]). Alternatively, fixing all SM parameters to their measured values and letting free $\alpha_{\mathrm{s}}$ in the full SM electroweak fit gives $\alpha_{\mathrm{s}}=0.1194 \pm 0.0029$ with a $\sim 2.4 \%$ uncertainty (blue curve in Fig. 2) [15].

At the FCC-ee, the availability of $10^{12} \mathrm{Z}$ bosons providing high-precision measurements with $\Delta m_{\mathrm{Z}}=0.1 \mathrm{MeV}, \Delta \Gamma_{\mathrm{Z}}^{\mathrm{tot}}=0.1 \mathrm{MeV}, \Delta \mathrm{R}_{\mathrm{Z}}=10^{-3}$ (achievable thanks to the possibility to perform a threshold scan including energy self-calibration with resonant depolarization) will reduce the $\alpha_{\mathrm{s}}\left(m_{\mathrm{z}}\right)$ uncertainty to $\sim 0.15 \%$. Figure 2 (right) shows the expected $\alpha_{\mathrm{s}}$ extractions from $\mathrm{R}_{\mathrm{Z}}$ and $\Gamma_{Z}^{\text {tot }}$ at the FCC-ee (yellow band) without theoretical uncertainties (dotted red curve) and with the theoretical uncertainties reduced to one-fourth of their current values (solid red curve) [15], a result 
that is $\sim 25$ times more precise than that from the current full SM electroweak fit today (blue band). Of course, since the main FCC-ee goal is to carry out "stress precision tests" of the SM in searches for physics beyond the SM, one would need to carefully compare the results of both extractions in order to identify possible deviations due to new physics (which, would potentially affect differently the result derived from the Z-pole data alone, and from the full SM fit).

\section{References}

[1] D. Buttazzo, G. Degrassi, P. P. Giardino, G. F. Giudice, F. Sala, A. Salvio and A. Strumia, JHEP 12 (2013) 089 [arXiv:1307.3536 [hep-ph]].

[2] D. d'Enterria, P. Z. Skands et al., arXiv:1512.05194 [hep-ph].

[3] C. Patrignani et al. [Particle Data Group], Chin. Phys. C 40 (2016) 100001.

[4] A. Blondel et al., arXiv:1905.05078 [hep-ph].

[5] P. A. Baikov, K. G. Chetyrkin and J. H. Kuhn, Phys. Rev. Lett. 101 (2008) 012002 [arXiv:0801.1821 [hep-ph]]; P. A. Baikov, K. G. Chetyrkin, J. H. Kuhn and J. Rittinger, Phys. Rev. Lett. 108 (2012) 222003 [arXiv:1201.5804 [hep-ph]].

[6] D. Kara, Nucl. Phys. B 877 (2013) 683 [arXiv:1307.7190 [hep-ph]].

[7] A. Freitas, JHEP 04 (2014) 070 [arXiv:1401.2447 [hep-ph]].

[8] I. Dubovyk, A. Freitas, J. Gluza, T. Riemann and J. Usovitsch, Phys. Lett. B 783 (2018) 86 [arXiv: 1804.10236 [hep-ph]].

[9] D. d'Enterria and M. Srebre, Phys. Lett. B 763 (2016) 465 [arXiv:1603.06501 [hep-ph]].

[10] M. Bicer et al. [TLEP Design Study Working Group], JHEP 01 (2014) 164; [arXiv:1308.6176 [hep-ex]]. D. d'Enterria, doi:10.1142/9789813224568_0028 [arXiv:1602.05043 [hep-ex]].

[11] A. Pich, A. Rodríguez-Sánchez, Phys. Rev. D 94 (2016) 034027 [arXiv:1605.06830 [hep-ph]].

[12] D. Boito, M. Golterman, K. Maltman and S. Peris, Phys. Rev. D 95 (2017) 034024; [arXiv:1611.03457 [hep-ph]]. S. Peris (2019), these proceedings.

[13] A. Denner, Fortsch. Phys. 41 (1993) 307 [arXiv:0709.1075 [hep-ph]].

[14] S. Schael et al. Phys. Rept. 427 (2006) 257 [hep-ex/0509008].

[15] J. Haller, A. Hoecker, R. Kogler, K. Mönig, T. Peiffer and J. Stelzer, Eur. Phys. J. C 78 (2018) 675 [arXiv: 1803.01853 [hep-ph]]. 